\title{
Erwerbstätigkeit im Alter und der Übergang in Rente - ein Überblick aus der Arbeitsweltberichterstattung
}

\author{
Arthur Kaboth, Susanne Drescher \& Martin Brussig
}

\section{Einleitung}

Die letzten Jahrzehnte waren in der Arbeitsmarkt- und Rentenpolitik von einem Paradigmenwechsel geprägt, durch den eine Umkehr von der Frühverrentung hin zur verlängerten Lebensarbeitszeit eingeleitet wurde. Die Ziele der sozialstaatlichen Alterssicherung - die Lebensstandardsicherung, die Vermeidung von (Alters-)Armut und die Milderung von Folgen erwerbsbedingter Risiken im Lebenslauf für die Alterssicherung - sind durch diesen Paradigmenwechsel, einhergehend mit dem Absinken des Rentenniveaus und der mehr oder weniger gescheiterten Umsetzung der privaten Altersvorsorge, gefährdet. Auch die Zukunftsfähigkeit der gesetzlichen Alterssicherung, insbesondere ihre Finanzierbarkeit, hängt von den Entwicklungen auf dem Arbeitsmarkt und der Erwerbsbeteiligung ab. Das Zusammenspiel von Arbeitsmarkt und Rente ist bereits Gegenstand vieler politischer und wissenschaftlicher Debatten geworden (Bäcker/Jansen/ Schmitz 2017; Brussig/Knuth/Mümken 2016; Brussig/Zink 2018); vor dem Hintergrund der sich verändernden Arbeitswelt, etwa durch die Digitalisierung, den großen Niedriglohnsektor und die Erosion des Normalarbeitsverhältnisses, ist es auch gegenwärtig wichtig, die Entwicklungen auf dem Arbeitsmarkt, im Sinne einer Arbeitsweltberichterstattung, kontinuierlich zu beobachten und auf (zukünftigen) Gestaltungsbedarf hinzuweisen.

Das vorliegende Kapitel bietet einerseits einen Überblick über die Entwicklungen in der Erwerbsbeteiligung Älterer. Diesbezüglich wird erläutert, wie es durch politische Reformen zu Veränderungen auf dem Arbeitsmarkt gekommen ist und daraus folgend, welche Entwicklungen es in der Beschäftigungsstruktur Älterer und im Rentenzugang gegeben hat. Darauf aufbauend werden andererseits Probleme und Herausforderungen identifiziert. Zudem wird die Erwerbsarbeit nach dem Renteneintritt betrachtet. Die für das Kapitel vorliegenden Ergebnisse und Erkenntnisse stammen 
vorwiegend aus dem Forschungsprojekt „Altersübergangs-Monitor“, einer kontinuierlichen Berichterstattung (seit 2004) zur Arbeitswelt und zum Altersübergang.

\section{Erwerbsbeteiligung im Alter}

Die Erwerbsbeteiligung Älterer ist seit der Jahrtausendwende stark gestiegen (siehe Abbildung 1). Hierin ist jede Art von Erwerbstätigkeit eingeschlossen, also auch selbstständige, geringfügige und Erwerbsarbeit in Teilzeit. Während in den 1990er Jahren die Erwerbsbeteiligung der 55-Jährigen (und älter) noch unter 40 Prozent lag, hat sie sich bis zum Jahr 2018 fast verdoppelt, sodass mehr als zwei Drittel aller Erwerbspersonen im Alter von 55 bis 64 Jahren erwerbstätig waren. Der Anstieg ab dem Jahr 2001 ist sowohl bei den Männern als auch bei den Frauen zu beobachten. Bei den älteren Frauen hat sich die Quote seit 1992 (23,3 Prozent) sogar nahezu verdreifacht (2018: 66,9 Prozent). Der Ursprung dieser Entwicklung lag in der Neuausrichtung der Beschäftigungs- und Rentenpolitik seit Anfang der 1990er Jahre, mit dem Ziel, einerseits die Beitragszeiten der Versicherten zu verlängern und andererseits die Rentenbezugszeiten zu verkürzen.

Abbildung 1: Erwerbstätigenquoten Älterer (55 bis 64 Jabre) nach Geschlecht, 1992 bis 2018

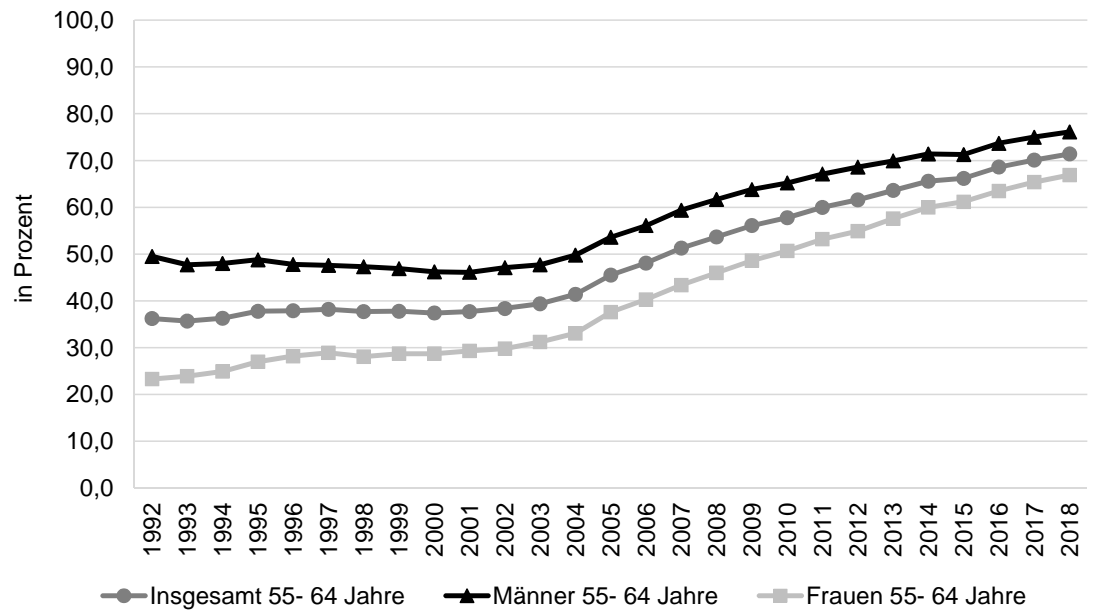

Quelle: Eurostat 2019, Labour-Force-Survey (LFS) [lfsa_epgaed]. Eigene Darstellung. 


\subsection{Rentenreformen zur verlängerten Lebensarbeitszeit}

Der Paradigmenwechsel in der Rentenpolitik wurde nicht erst mit der Einführung der „Rente mit 67“ eingeleitet, sondern bereits mit dem Rentenreformgesetz von 1989, das in wesentlichen Teilen 1992 in Kraft getreten ist. Mit diesem Gesetz wurden ab dem Jahr 2001 die Altersgrenzen von vorzeitig beziehbaren Altersrenten auf das Niveau der Regelaltersgrenze von 65 Jahren angehoben, sowie Abschläge bei vorzeitigen Renteneintritt eingeführt (Schmähl 2011a). Ebenfalls im Jahr 2001 löste die Erwerbsminderungsrente ${ }^{1}$ die bisherige Berufs- und Erwerbsunfähigkeitsrente ab, wobei die Erwerbsfähigkeit allein maßgeblich für den Leistungsbezug wurde. Mit dem erwerbsorientierten Konzept wird geprüft, ob und in welchem Maße die betroffene Person einer Erwerbstätigkeit jeglicher Art auf dem allgemeinen Arbeitsmarkt nachgehen kann (Bäcker 2012). Im Jahr 2007 folgte schließlich das Altersgrenzenanpassungsgesetz, welches die stufenweise Heraufsetzung der Altersgrenze auf das 67. Lebensjahr ab 2012 beinhaltete. Zeitgleich, das heißt ab 2012, endete der Zugang in die Altersrente für Frauen und die Altersrente nach Altersteilzeitarbeit bzw. wegen Arbeitslosigkeit. Diese Möglichkeiten der Frühverrentung wurden für Personen, die nach 1952 geboren sind, geschlossen. Der Altersübergang wurde somit entflexibilisiert. Darüber hinaus wurde die Altersrente für besonders langjährig Versicherte (45 Jahre Versicherungszeit und ohne Abschläge) neu eingeführt (Schmähl 2011b) und anschließend im Jahr 2014 (Leistungsverbesserungsgesetz ${ }^{2}$ ) reformiert, sodass das Renteneintrittsalter dieser Altersrente vorübergehend auf 63 Jahre herabgesetzt wurde. Aber auch hier wird das Renteneintrittsalter wie bei der Regelaltersrente stufenweise bis auf 65 Jahre angehoben (Dünn/Stosberg 2014). Mit dem Flexirentengesetz (in Kraft seit 2017) wurde der Altersübergang schließlich reflexibilisiert, worauf in Kapitel 6 näher eingegangen wird.

1 Gesetz zur Reform der Renten wegen verminderter Erwerbsfähigkeit.

2 Das Leistungsverbesserungsgesetz der Rentenversicherung beinhaltete zudem die Aufstockung von Kindererziehungszeiten für vor 1992 geborene Kinder („Mütterrente“), die Erweiterung der Zurechnungszeit um zwei Jahre in der Erwerbsminderungsrente und die Erhöhung des Budgets für Rehabilitationsleistungen. 


\subsection{Arbeitsmarktreformen}

Gegenüber der Rentenpolitik blieb die Neuausrichtung der Arbeitsmarktpolitik in ihrer Absicht zur Verlängerung der Lebensarbeitszeit und zur Bewältigung des demografischen Wandels eher ambivalent (Brussig/ Knuth/Mümken 2016). Dennoch können einzelne Veränderungen in der Arbeitsmarktpolitik als Gründe für die Zunahme der Alterserwerbsbeteiligung und als Reformen zur Umkehr der Frühverrentung eingeschätzt werden.

Nahezu zeitgleich mit der Zusammenführung der Arbeitslosen- und Sozialhilfe in das neue Leistungssystem zur Grundsicherung für Arbeitsuchende (SGB II/„Hartz IV“) im Jahr 2005 wurde mit dem Gesetz zu Reformen am Arbeitsmarkt die Bezugsdauer des Arbeitslosengeldes (SGB III) insbesondere für Ältere drastisch verkürzt, in den Folgejahren aber wieder moderat verlängert. ${ }^{3}$ Nach der Gesetzesänderung gingen die Eintrittsraten in Arbeitslosigkeit von Älteren deutlich zurück; Arbeitslosigkeit galt bei Älteren häufig als „Brücke“ in den Ruhestand (Dlugosz/Stephan/Wilke 2009; Knuth/Kalina 2002). Als weiterer Baustein zur Abkehr von den Frühverrentungen in der Beschäftigungspolitik wird das Auslaufen der geförderten Altersteilzeit im Jahr 2009 gesehen. Die überwiegend im Blockmodell (Beschäftigungs-, dann Freistellungszeit) genutzte Altersteilzeit galt als Möglichkeit des geförderten Vorruhestands. Dies stand dem Leitbild eines gleitenden Rentenübergangs über eine schrittweise Reduzierung der Arbeitszeit entgegen (Brussig/Knuth/Wojtkowski 2009; Wanger 2009).

Die Förderung von älteren Arbeitslosen im SGB II wurde durch die Umgestaltung zur aktivierenden Arbeitsmarkpolitik verankert und gleichzeitig durch Bundesprogramme unterstützt. Durch die Initiative „Perspektive 50plus“" (von 2005 bis 2015) wurden Langzeitarbeitslose im SGB II ab dem 50. Lebensjahr durch individuelle und intensive Betreuung auf dem ersten Arbeitsmarkt integriert. Dabei zeigte sich, dass die Integrationsquoten der Geförderten höher und die Kosten der Förderung niedriger ausfielen als die der Regelförderung (Knuth/Stegmann/Zink 2014). Das Programm zur Weiterbildung Geringqualifizierter und beschäftigter Älterer in Unternehmen (WeGebAU) hat das Ziel, die Lebensarbeitszeit zu verlängern und zugleich die Arbeitslosigkeit Älterer zu vermeiden, in dem in die Qualifikation dieser Personengruppen investiert wird. Untersuchungen zu diesen Programmen belegen, dass sich die präventiven Maßnahmen posi-

3 Ab dem Jahr 2008 wurde die maximale Bezugsdauer bei Arbeitslosen ab 58 Jahren wieder leicht angehoben (von 18 auf 24 Monate). 
tiv auf den Verbleib in sozialversicherungspflichtiger Beschäftigung ausgewirkt haben (Singer 2013). Allerdings investieren überwiegend mittlere und große Unternehmen und seltener Kleinbetriebe in die Qualifikationen ihrer Belegschaft (Lott 2011).

Der Paradigmenwechsel in der Rentenpolitik begann früher als in der Arbeitsmarktpolitik und konzentrierte sich darauf, die Erwerbsphasen zu verlängern, vorwiegend um die (zukünftige) Finanzierung der Gesetzlichen Rentenversicherung über das Umlageverfahren zu gewährleisten. In der Arbeitsmarktpolitik setzte der Paradigmenwechsel hingegen erst mit dem Beginn der Jahrtausendwende ein. Dieser Wechsel fällt zudem weniger eindeutig aus als in der Rentenpolitik, insbesondere weil im SGB II Sonderregelungen für Ältere erhalten geblieben sind, die der Verlängerung der Lebensarbeitszeit entgegenwirken. Dennoch zeigen die angeführten Regelungen und Programme der Arbeitsmarktpolitik durchaus Elemente, die auf eine verlängerte Erwerbsteilhabe zielen bzw. diese unterstützen.

\subsection{Qualifikationsspezifische Erwerbsbeteiligung}

Neben der Neuausrichtung der Beschäftigungs- und Rentenpolitik ist die Zunahme der Alterserwerbsbeteiligung auf weitere Faktoren zurückzuführen, welche in komplexen Wechselwirkungen zueinander stehen. Hervorzuheben ist diesbezüglich das Qualifikationsniveau bzw. die zunehmend höheren Bildungsabschlüsse der Beschäftigten; diese Entwicklung resultiert im Wesentlichen, und vor allem bei Frauen, aus der Bildungsexpansion in den 1970er und 1980er Jahren (Ziefle 2017).

In der Abbildung 2 werden die qualifikationsspezifischen Erwerbstätigenquoten der 25- bis 54-Jährigen (auf der X-Achse) denen der Personen im Alter von 55 bis 64 Jahren (auf der Y-Achse) im Vergleich der Jahre 2006 zu 2018 gegenübergestellt. Die abgebildete Diagonale wird als Alterslücke bezeichnet. Liegt einer der Datenpunkte auf dieser Diagonalen, bedeutet dies, dass die Quoten der Altersgruppen gleich groß sind.

In Abbildung 2 wird deutlich, dass die Erwerbsbeteiligung sowohl bei der jüngeren als auch bei der älteren Altersgruppe vom Qualifikationsniveau abhängt. Personen mit niedriger Qualifikation weisen geringere Erwerbstätigenquoten auf als Personen mit einem hohen Bildungsabschluss. Qualifikationsübergreifend sind die Quoten der Älteren niedriger als die der Personen im Haupterwerbsalter (25-54 Jahre), allerdings hat sich die Alterslücke zwischen beiden Altersgruppen zwischen 2006 und 2018 nahezu geschlossen. Die Erwerbschancen nehmen mit steigender Qualifikation 
zu und sind, gemessen an der Alterslücke, vor allem bei Älteren mit mittlerem Qualifikationsabschluss noch nicht vollständig ausgeschöpft. Das Qualifikationsniveau kann jedoch kein eigenständiger Faktor zur Erklärung der Zunahme der Alterserwerbsbeteiligung darstellen, denn auch unter den Geringqualifizierten sind die Quoten unter den Älteren beachtenswert gestiegen.

\section{Abbildung 2: Erwerbstätigenquoten und Alterslücke nach Bildungsabschluss} und Altersgruppen im Vergleich 2006 und 2018

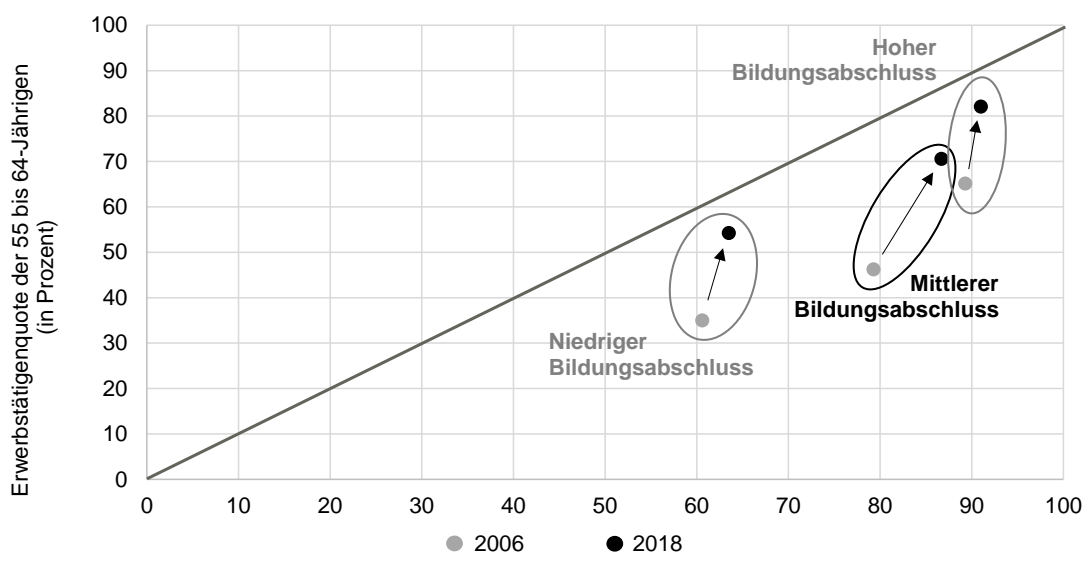

Erwerbstätigenquote der 25 bis 54-Jährigen (in Prozent)

Quelle: Eurostat 2019 (LFS) [lfsa_ergaed]. ISCED11 Kategorien nach Eurostat (2019): Niedriger Bildungsabschluss ISCED Stufen 0-2: Bis zu Hauptabschluss; Mittlerer Bildungsabschluss ISCED Stufen 3-4: Oberstufe/Berufsausbildung; Hoher Bildungsabschluss ISCED Stufen 5-8: Akademische Abschlüsse. Eigene Darstellung.

\subsection{Demografie als Ursache?}

Die demografische Entwicklung wird oft als Ursache für die finanzielle Belastung der gesetzlichen Alterssicherung angesehen. Das Verhältnis von Beitragszahlenden zu Empfänger*innen von Leistungen verschiebt sich und wird sich mit den beginnenden Renteneintritten der geburtenstarken „Babyboomer" Generation (1955 bis 1969) weiter verschieben. Allerdings bleibt häufig unberücksichtigt, dass die demografische Entwicklung auch zur Zunahme der Alterserwerbsbeteiligung, zumindest vorübergehend, 
beigetragen hat. Vor allem nach der Jahrtausendwende gab es in Deutschland einen demografischen „Rückenwind“ (Mümken/Brussig 2012), aufgrund dessen sich die Erwerbsbeteiligung Älterer erhöht hat. Er resultierte daraus, dass in der Altersgruppe der 55- bis 64-Jährigen die jüngeren Jahrgänge (mit altersbedingt hoher Erwerbsbeteiligung) zahlenmäßig stärker waren als die älteren Jahrgänge (mit niedriger Erwerbsbeteiligung). Im Vergleich zu anderen europäischen Ländern ist der demografische Effekt in Deutschland im Zeitraum zwischen 2002 und 2008 besonders hoch (Brussig/Wojtkowski 2008; Mümken/Brussig 2012).

\subsection{Stabile Wirtschaftsentwicklung und langer Verbleib Älterer in Beschäftigung}

Die stabile Wirtschaftsentwicklung seit 2006, gemessen am Bruttoinlandsprodukt (BIP), hat ebenso zu der Zunahme der Erwerbsbeteiligung Älterer beigetragen, denn eine positive Konjunktur schafft neue und sichert bestehende Arbeitsverhältnisse. Aber auch in diesem Zusammenhang spielen Wechselwirkungen mit weiteren Faktoren eine Rolle, wie die jüngste Finanz- und Wirtschaftskrise zeigte. Zunächst wird aus Abbildung 3 ersichtlich, dass (1.) das BIP mit dem Ausgangsjahr 2005 kontinuierlich, mit Ausnahme der Finanz- und Wirtschaftskrise zwischen 2009 und 2010, gestiegen ist und (2.) die Alterslücke ${ }^{4}$, unbeeindruckt von der wirtschaftlichen Entwicklung, auch in Zeiten der Krise kleiner geworden ist (Kaboth/Brussig 2018).

Diese Entwicklung - schrumpfende Alterslücke trotz Wirtschaftskrise stellt ein Novum in der Alterserwerbsbeteiligung dar, denn in früheren Rezessionen der 1970er, 1980er oder 1990er Jahre ist die Beteiligung Älterer am Arbeitsmarkt gesunken und wurde überwiegend durch Frühverrentungen organisiert (OECD 2013). Frühverrentungen sind rentenrechtlich nicht mehr möglich und mithin für die Unternehmen deutlich teurer als in der Vergangenheit (Brussig/Knuth/Wojtkowski 2009); sie stellen einen wesentlichen Grund für die sinkende Alterslücke dar. Zugleich haben betriebliche und staatliche Maßnahmen während der Krise zur stabilen Erwerbsbeteiligung Älterer beigetragen, beispielsweise durch die Reduzierung von Arbeitszeit mit Hilfe von Kurzarbeit, Arbeitszeitkonten oder Be-

4 Die Alterslücke beschreibt die Differenz der Erwerbstätigenquoten (in Prozent) zwischen den Älteren ( 55 bis 64 Jahre) und denen im Haupterwerbsalter (15 bis 54 Jahre). 
triebsvereinbarungen (Stein/Aricò 2010). Die hohe Partizipation Älterer am Arbeitsmarkt resultiert vorwiegend aus dem längeren und ununterbrochenen Verbleib in Beschäftigung und nur zu einem kleineren Anteil aus Neueinstellungen (Brussig/Eggers 2014). Insgesamt haben aber auch die Neueinstellungen Älterer kontinuierlich zugenommen, allerdings ist das Arbeitskräftepotenzial noch nicht ausgeschöpft und auch die Rekrutierungsstrategien der Betriebe haben sich weitestgehend noch nicht an die demographischen Gegebenheiten angepasst (Brussig 2011). Des Weiteren sind die Eintrittsraten in Beschäftigung in den Beschäftigungsbereichen hoch, wo die Beschäftigungsstabilität niedrig ist, insbesondere bei Frauen und Teilzeitbeschäftigten (Brussig/Eggers 2014).

Abbildung 3: Entwicklung des Bruttoinlandsprodukts (Index, linke Achse) und der Alterslücke (in Prozent, rechte Achse) 2005 bis 2016

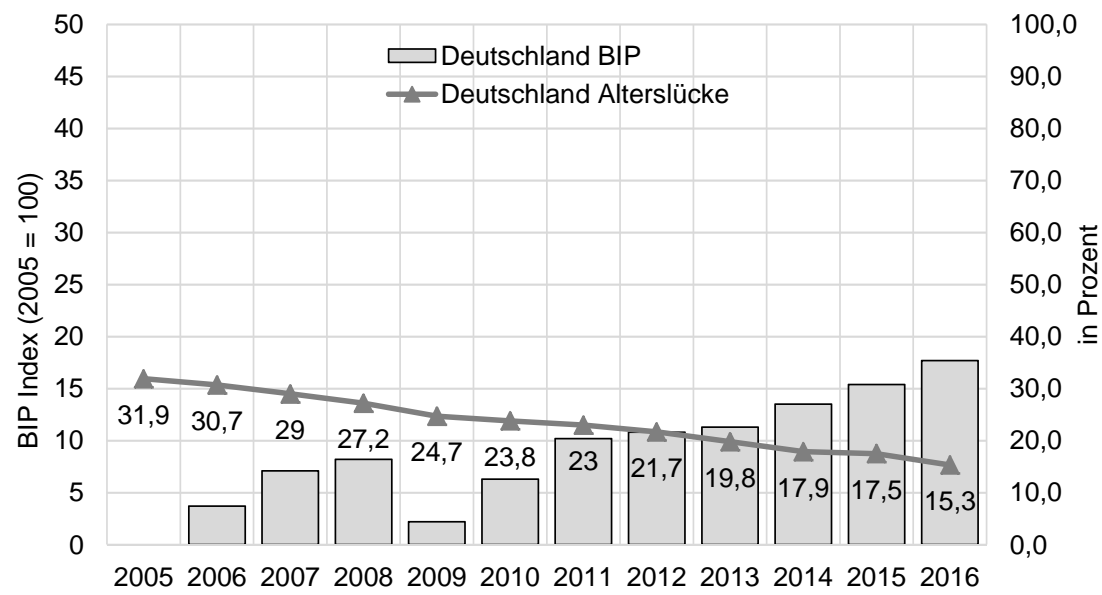

Quelle: Kaboth \& Brussig (2018). Eigene Darstellung.

\subsection{Struktur der Beschäftigung}

Allein eine Erwerbsteilhabe ist jedoch nicht mit einer ausreichenden Absicherung der Beschäftigten im Alter gleichzusetzen. Denn ein Rentenanspruch in der gesetzlichen Alterssicherung ergibt sich nicht nur aus der Dauer der Erwerbsphase, sondern auch aus dem im Erwerbsleben erzielten 
Einkommen. So können besonders viele oder längere Phasen in Teilzeitbeschäftigung zu niedrigen Renten aus dem gesetzlichen Sicherungssystem führen (Brussig/Zink 2018).

Tabelle 1: Anteile (in Prozent) und Anzahl (in Tausend) von Vollzeit- und Teilzeitbeschäftigung an allen Beschäftigten nach Geschlecht im Alter von 55 bis 64 Jahren, 2000 bis 2018

\begin{tabular}{rrrrrrrrrr}
\hline & & 2000 & & 2006 & & 2012 & & 2018 \\
\hline \multirow{2}{*}{ Männer } & Vollzeit & $93,3 \%$ & $(2.442,9)$ & $89,4 \%$ & $(2.395,8)$ & $89,0 \%$ & $(3.081,9)$ & $89,5 \%$ & $(4.025,1)$ \\
& Teilzeit & $6,7 \%$ & $(176,0)$ & $10,6 \%$ & $(283,2)$ & $11,0 \%$ & $(379,9)$ & $10,5 \%$ & $(470,7)$ \\
\multirow{2}{*}{ Frauen } & Vollzeit & $51,7 \%$ & $(846,2)$ & $48,0 \%$ & $(943,5)$ & $49,4 \%$ & $(1.439,7)$ & $48,1 \%$ & $(1.939,6)$ \\
& Teilzeit & $48,3 \%$ & $(790,7)$ & $52,0 \%$ & $(1.021,7)$ & $50,6 \%$ & $(1.472,6)$ & $51,9 \%$ & $(2.094,9)$ \\
\hline
\end{tabular}

Quelle: Eurostat 2019 (LFS) [lfsa_epgaed]. Eigene Darstellung.

Vor dem Hintergrund der gestiegenen Alterserwerbsbeteiligung wird in Tabelle 1 die Entwicklung der Beschäftigungsstruktur unter den Älteren ab dem 55. Lebensjahr dargestellt. Sowohl bei Männern als auch bei Frauen hat der Anteil in Teilzeitbeschäftigung zugenommen, allerdings auf unterschiedlichem Niveau. Der Anteil älterer Männer in Teilzeitbeschäftigung ist von 6,7 Prozent im Jahr 2000 bis zum Jahr 2018 auf 10,5 Prozent angewachsen. Diese Entwicklung geht dabei mit einer deutlichen Zunahme sowohl der in Teilzeit als auch der in Vollzeit beschäftigten Männer einher. Die Anzahl der in Teilzeit- und Vollzeit beschäftigten Frauen steigt von 2000 bis 2018 sogar noch wesentlich stärker, wenngleich sich die Verteilung von Teilzeit und Vollzeit nur wenig ändert. Bei den älteren Frauen lag der Anteil der Teilzeitbeschäftigung an allen Beschäftigten bereits im Jahr 2000 bei 48,3 Prozent und steigerte sich bis zum Jahr 2018 auf 51,9 Prozent, sodass jede zweite Frau im Alter von 55 bis 64 Jahren in Teilzeit beschäftigt war.

Der generelle Anstieg der voll- und teilzeitbeschäftigten Älteren veranschaulicht einen Wandel in der Arbeitswelt: Immer mehr Ältere sind beschäftigt, insbesondere immer mehr ältere Frauen. Zugleich geht damit eine Verbreitung von Teilzeitbeschäftigung einher. Dies kann allerdings mit einem Nachteil für die Alterssicherung verbunden sein, da durch eine dauerhafte Teilzeitbeschäftigung nur geringe Rentenansprüche erworben werden können. In der Folge besteht ein höheres (Alters-) Armutsrisiko, dem dann insbesondere Frauen ausgesetzt sind.

Da die Bildung als ein wesentlicher Faktor der Alterserwerbsbeteiligung im vorherigen Kapitel identifiziert wurde, wird sie hier im Rahmen der Beschäftigungsstruktur wieder aufgegriffen (Abbildung 4). Dabei wird deutlich, dass der Bildungsabschluss bei den Männern kaum Auswirkun- 
gen auf die Beschäftigungsstrukturen der Jahre 2006, 2012 und 2018 hat. Bei den Frauen hingegen sind die Anteile mit mittlerer Qualifikation in Teilzeitbeschäftigung höher als in Vollzeit. Im Beobachtungszeitraum haben diese Anteile sogar erheblich zugenommen, sodass der Zuwachs der Erwerbsbeteiligung bei den älteren Frauen überwiegend auf diejenigen mit mittlerer Qualifikation in Teilzeit zurückzuführen ist. Zudem scheint ein hoher Bildungsabschluss bei den Frauen die Wahrscheinlichkeit, in einer Vollzeitstelle beschäftigt zu sein, sichtlich zu erhöhen.

\section{Abbildung 4: Beschäftigung in Voll-und Teilzeit nach Geschlecht und Bildungs-} abschluss der 55 bis 64-Jäbrigen, 2006 bis 2018

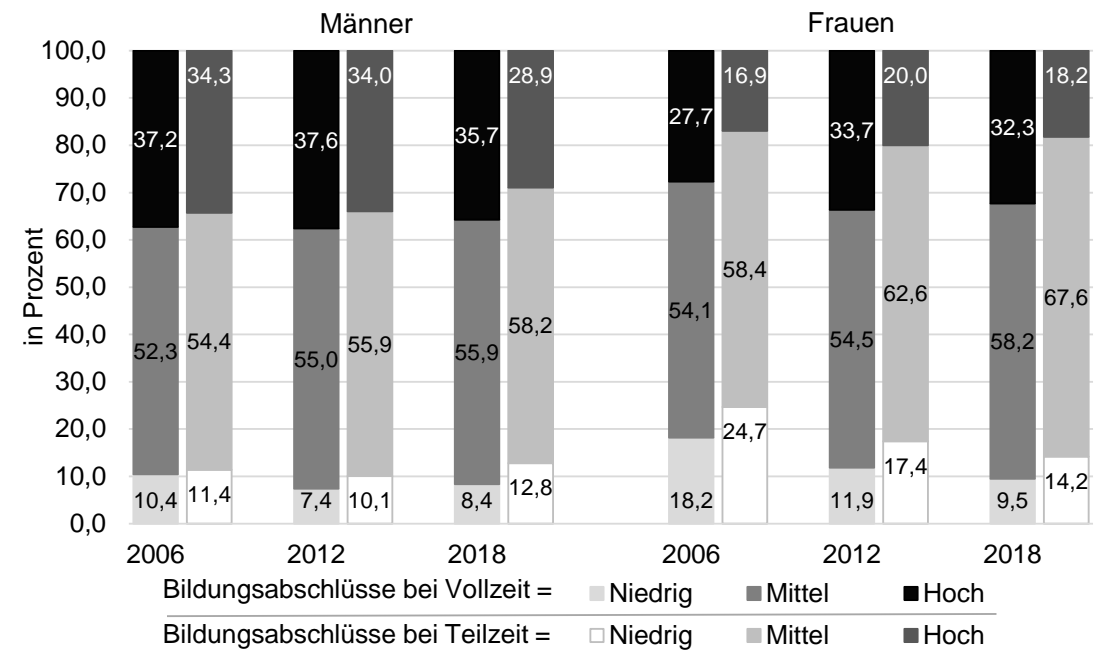

Quelle: Eurostat 2019 (LFS) [lfsa_epgaed]; ISCED11 Kategorien nach Eurostat (2019): Niedriger Bildungsabschluss ISCED Stufen 0-2: Bis zu Hauptabschluss; Mittlerer Bildungsabschluss ISCED Stufen 3-4: Oberstufe/Berufsausbildung; Hoher Bildungsabschluss ISCED Stufen 5-8: Akademische Abschlüsse. Eigene Darstellung.

\section{Altersarbeitslosigkeit}

Neben einer durch (dauerhafte) Teilzeit geprägte Beschäftigung können (längere) Phasen von Arbeitslosigkeit das Risiko einer unzureichenden Alterssicherung erhöhen, nicht zuletzt da seit 2011 keine Pflichtbeiträge mehr für ALG-II-Beziehende in der Rentenversicherung erbracht werden 
(Mika/Lange/Stegmann 2014). Für Ältere stellt Arbeitslosigkeit ein besonderes Risiko hinsichtlich der gesetzlichen Alterssicherung dar: Auch wenn unter den Älteren die Wahrscheinlichkeit arbeitslos zu werden gering ist, ist die Wahrscheinlichkeit arbeitslos zu bleiben besonders hoch. Die Wiedereinstellungschancen unter den Älteren sind also sehr gering (Bundesagentur für Arbeit 2018). Ältere Arbeitslose können demnach besonders von kumulierten Dauern in Arbeitslosigkeit betroffen sein. Zudem garantiert eine Zunahme der Alterserwerbsbeteiligung keine Abnahme der Altersarbeitslosigkeit, denn die Anzahl der Arbeitslosen kann zeitgleich steigen, wenn die Anzahl an Nichterwerbspersonen sinkt (Kaboth/Brussig 2020; Mümken/Brussig 2013).

Abbildung 5: Arbeitslosenquoten nach Altersgruppen, 2009 bis 2018

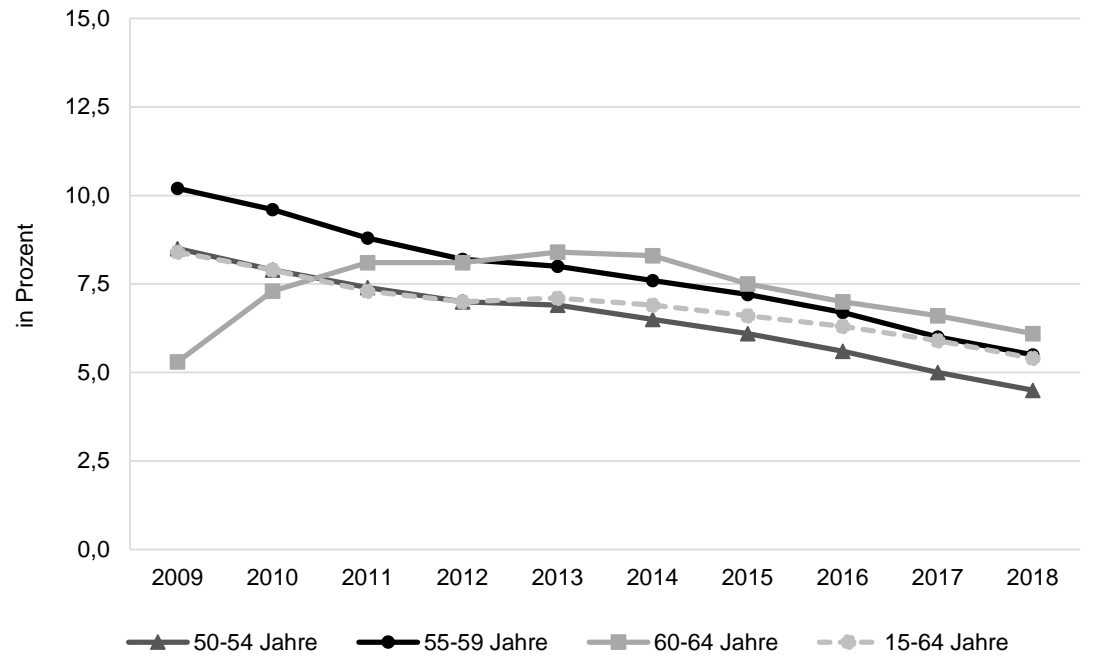

Quelle: Sonderauswertung der Bundesagentur für Arbeit 2019. Eigene Darstellung.

Die Entwicklung der Arbeitslosigkeit unter den Älteren (siehe Abbildung 5) verdeutlicht, dass mit zunehmendem Alter die relative Betroffenheit von Arbeitslosigkeit zunimmt: Die Arbeitslosenquoten der 60- bis 64-Jährigen sind seit 2013 unter den in der Grafik dargestellten Altersgruppen am höchsten. Zurückzuführen ist diese Entwicklung insbesondere auf die Schließung der Frühverrentungsmöglichkeiten, in denen der Renteneintritt - wenn auch mit Abschlägen - bereits mit 60 Jahren erfolgen konnte. 
Aufgrund des inzwischen frühestmöglichen Renteneintritts erst mit 63 Jahren und der stufenweisen Anhebung der Regelaltersgrenze, hat sich die Arbeitslosigkeit bis ins höhere Erwerbsalter ausgeweitet.

Dabei werden die Arbeitslosenquoten, vor allem bei den 60 bis 64-Jährigen, noch deutlich unterschätzt, denn nach wie vor ist eine vorruhestandsähnliche gesetzliche Regelung in Kraft. Nach dieser Regelung wird es Älteren im SGB II ermöglicht, unter erleichterten Bedingungen Leistungen zu beziehen, auch ohne einer Arbeitsvermittlung, wie sonst erforderlich, zur Verfügung stehen zu müssen. Diese Sonderregelung für Ältere nach $\ 53 \mathrm{a}$ Abs. 2 SGB II schließt Personen ab dem 59. Lebensjahr aus der Arbeitslosenstatistik aus, die unter Bezug von Leistungen innerhalb der letzten zwölf Monate keine sozialversicherungspflichtige Beschäftigung oder eine Maßnahme der Arbeitsförderung seitens der Arbeitsvermittlung angeboten bekommen haben. Seit dem Jahr 2015 gelten deshalb jährlich etwa 140.000 Personen im Alter von 60 bis 64 Jahren nicht als arbeitslos und bleiben verdeckt. Zum Vergleich: Insgesamt waren im Jahr 2018 ca. 210.000 Personen in dieser Altersgruppe offiziell arbeitslos (Kaboth/Brussig 2020). Zudem wurden ältere Arbeitslose ab dem 55. Lebensjahr im Vergleich zu allen Altersgruppen zwischen 2010 und 2015 unterdurchschnittlich gefördert, vor allem hinsichtlich qualifizierender Maßnahmen (Walwei 2018).

\section{Renteneintritt}

Der Rentenzugang spiegelt kurzfristig wie langfristig wirksame rentenrechtliche Änderungen wider. Die kurzfristig wirksamen Änderungen haben in der Anzahl an Rentenzugängen und in dem deutlichen Anstieg des durchschnittlichen Rentenzugangsalters in Altersrenten vor allem in den Jahren 2014 und 2015 ihren Niederschlag gefunden. Verantwortlich für diese Entwicklung war zum einen die Anrechnung eines zweiten Entgeltpunktes für jedes vor 1992 geborene Kind („Mütterrente“) nach dem RVLeistungsverbesserungsgesetz. Durch diese Regelung erwarben einige Personen, überwiegend westdeutsche Frauen, erstmals eine Rentenzugangsberechtigung. Zum anderen konnten durch die Reformierung der Altersrente für besonders langjährig Versicherte überwiegend Männer, die die Versicherungszeit von 45 Jahren erfüllten, abschlagsfrei in Rente gehen, da das Rentenzugangsalter vorübergehend auf 63 Jahre herabgesetzt wurde und diese Rente bei ihrer Einführung rückwirkend häufig im Alter von 64 Jahren beansprucht wurde (Kaboth/Brussig 2019). 
Aber auch die langfristigen Veränderungen im Rentenrecht, beispielweise das Auslaufen frühzeitig beziehbarer Altersrenten, spiegeln sich im Renteneintritt der Versicherten wider. So ist das durchschnittliche Renteneintrittsalter bei den Altersrenten bis 2013, zumindest bei den Männern, kontinuierlich gestiegen. Nach 2013 stagniert das Renteneintrittsalter trotz steigender Altersgrenzen, wenn der Einmaleffekt der „Mütterrente“ herausgerechnet wird, und beträgt im Jahr 2017 bei den Frauen 64,4 und bei den Männern 64,3 Jahre. Im Vergleich zum Jahr 2004 ist dies ein Zuwachs von mehr als einem Jahr, sowohl bei Männern als auch bei Frauen (Kaboth/Brussig 2019).

\section{Abbildung 6: Prozentualer Anteil der Altersrentenarten an allen Altersrenten-} zugängen nach Geschlecht, 2004 bis 2017

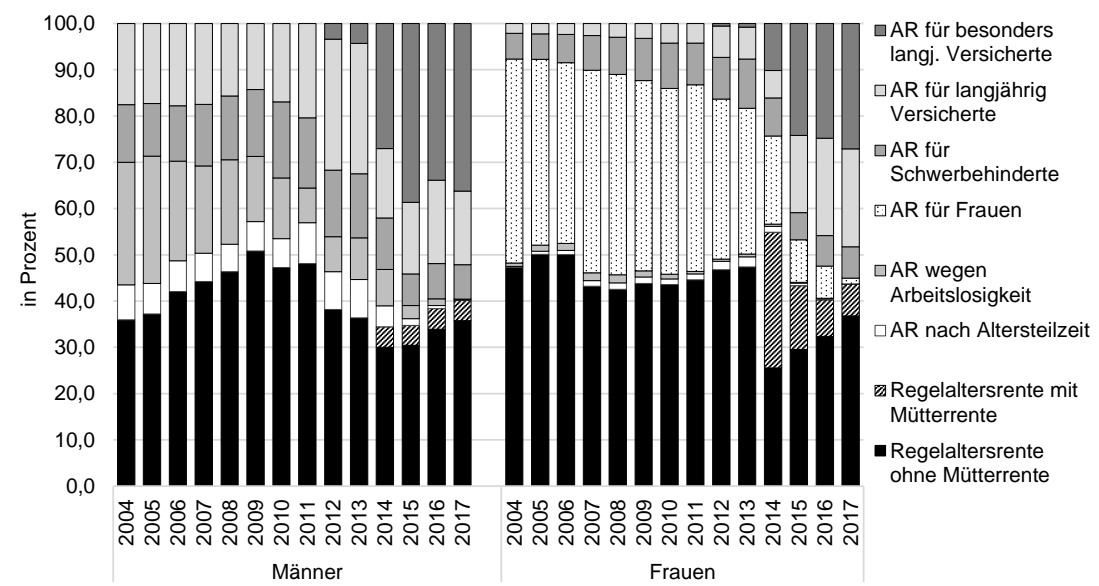

Quelle: Kaboth \& Brussig (2019).

Sowohl die langfristigen als auch die kurzfristigen Umgestaltungen schlagen sich im Zugang in unterschiedlichen Rentenarten nieder (siehe Abbildung 6). Insbesondere betrifft dies die Regelaltersrente, in der die stufenweise Anhebung der Regelaltersgrenze auf 67 Jahre ab der Geburtskohorte 1964 abgeschlossen sein wird. Die Zugänge in die Regelaltersrente sind bei den Männern seit 2011 und bei den Frauen seit 2015 rückläufig, weil die vorzeitig beziehbaren Altersrenten, auch mit Abschlägen, häufig in Anspruch genommen wurden. Inzwischen ist die Altersrente für langjährig Versicherte der frühestmögliche Rentenzugang (mit 63 Jahren) bei den Altersrenten. Allerdings steigt hier das abschlagsfreie Zugangsalter, wie in der Regelaltersgrenze stufenweise bis auf 67 Jahre an, sodass für den vor- 
zeitigen Renteneintritt die Abschläge auf bis zu 14,4 Prozent zunehmen werden. Diesbezüglich bleibt abzuwarten, wie die Versicherten, die die Beitragszeiten von 45 Jahren (Altersrente für besonders langjährig Versicherte) nicht erfüllen, auf die zunehmenden Abschläge beim frühestmöglichen Rentenzugang reagieren werden, und ob der Zeitpunkt für den Renteneintritt aufgrund von zunehmenden Abschlägen aufgeschoben wird.

Der Renteneintritt kann sowohl freiwillig als auch unfreiwillig erfolgen. Bis zum Jahresende 2016 konnten ältere Beziehende von Arbeitslosengeld II vonseiten der Jobcenter verpflichtet werden, vorzeitig eine Rente zu beantragen, auch wenn diese mit Abschlägen behaftet ist. Seit der Unbilligkeitsverordnung, die zum Jahresbeginn 2017 in Kraft trat, kann eine Verpflichtung zum Renteneintritt nur dann erfolgen, wenn für die betroffenen Personen keine Hilfebedürftigkeit im Alter entstehen würde (BMAS 2016). Die Unbilligkeitsverordnung ändert nichts daran, dass Menschen ihren erarbeiteten Rentenanspruch um die Höhe der Abschläge kürzen müssen. Dabei kann mit quantitativen Daten nur begrenzt nachgewiesen werden, ob der Renteneintritt selbstbestimmt ist und inwiefern eine Abschaffung der verpflichtenden Inanspruchnahme einer Altersrente auch mit Abschlägen eine Verhaltensänderung hinsichtlich eines späteren Renteneintritts bei den Beziehenden von Arbeitslosengeld II bewirken würde (Brussig 2015b).

\section{Arbeit im Rentenalter}

Erwerbstätigkeit im Alter endet nicht zwangsläufig mit dem Eintritt in die Rente. Die Anzahl von Personen, die neben ihrer Rente einer Erwerbstätigkeit nachgehen, hat im letzten Jahrzehnt kontinuierlich zugenommen. So waren im Jahr 2015 über 1 Mio. Personen im Alter von 65 Jahren und älter erwerbstätig (Bäcker/Jansen/Schmitz 2017)..$^{5}$ Von Interesse sind vor allem die Motive der Erwerbstätigkeit, die Tätigkeiten und die Beschäftigungsstruktur der erwerbstätigen Rentner*innen. Aus der Forschungsliteratur wird ersichtlich, dass es sich bei den arbeitenden Rentner*innen häufig um geringfügig Beschäftigte mit mindestens einer mittleren Qualifikation handelt, die überwiegend einfache Tätigkeiten ausüben. Nahezu ebenso häufig sind Selbstständige noch über die Regelaltersgrenze hinaus

5 Zu berücksichtigen ist, dass 2015 die Regelaltersgrenze bei 65 Jahren und vier Monaten lag. Somit sind in die Berechnung auch Personen eingegangen, die noch keine Altersrente beziehen können. 
erwerbstätig. Die Motive für die Erwerbstätigkeit der Rentner*innen sind vielfältig und erstrecken sich von finanzieller Notwendigkeit bis hin zu sozialer Teilhabe. Dabei ist zu berücksichtigen, dass sich die materiellen und immateriellen Motive nicht gegenseitig ausschließen, sondern häufig auch ergänzen (Bäcker/Jansen/Schmitz 2017). Um die Entwicklungen der Weiterarbeit Älterer weiter zu fördern, aber auch im Allgemeinen den Altersübergang zu reflexibilisieren, wurde die Teilrente mit dem Flexirentengesetz (seit 2017 in Kraft) reformiert (Deutscher Bundestag 2016). Welche Wirkung diese Reform auf die Erwerbsbeteiligung im Rentenalter hat, bleibt abzuwarten. Überwiegend erfolgt die Weiterbeschäftigung aus immateriellen Motiven, die allerdings nicht als Argument für eine weitere Anhebung der Regelaltersgrenze eingebracht werden sollte, denn die Erwerbstätigkeit als Rentner"in ist voraussetzungsvoll: Es handelt sich mehrheitlich um Rentner*innen mit hoher Qualifikation, gutem Gesundheitszustand und geringen Arbeitsbelastungen. Jedoch dürften bei Weitem nicht alle Rentner*innen diese Voraussetzungen erfüllen (Bäcker/Jansen/ Schmitz 2017).

\section{Fazit: Anforderungen an die Politik, Tarifpartner und Betriebe}

Die vorliegenden Ergebnisse verdeutlichen, dass die Bildungsexpansion, die wirtschaftliche Entwicklung und vor allem die Neuausrichtung der Beschäftigungs- und Rentenpolitik zur Zunahme der Alterserwerbsbeteiligung beigetragen haben. Zugleich sind allerdings auch Kehrseiten dieser Entwicklung zu beobachten: Hohe Teilzeitbeschäftigung bei älteren Frauen, die Ausweitung der Arbeitslosigkeit bis ins höhere Erwerbsalter und die geringen Neueinstellungen Älterer. Auch aufgrund dieser Entwicklungen auf dem Arbeitsmarkt erfolgen Renteneintritte vor der Regelaltersgrenze und belegen, dass eine (weitere) Heraufsetzung des Rentenalters nicht zwangsläufig zu verlängerten Erwerbsbiografien führt.

Handlungsbedarfe bestehen in der Reduzierung und Vermeidung von Arbeitslosigkeit sowie in der Förderung von Qualifikationen. Im Hinblick auf den Arbeitszeitumfang muss eine lebenslauforientierte Arbeitszeitgestaltung angestrebt werden, die sowohl den Anspruch nach Vereinbarkeit mit dem Privatleben erfüllt, als auch Möglichkeiten zur Belastungsreduktionen im Alter bietet, ohne dass dadurch die Gefahr der Altersarmut steigt. Des Weiteren sollten die Sonderregelung für Ältere nach SGB II und die Zwangsverrentung abgeschafft werden, die der Verlängerung des Erwerbslebens entgegenwirken und deren Durchsetzung nicht zum Kernaufgabenbereich der Arbeitsvermittlung gehören. Weitere wichtige Stell- 
schrauben, um die steigenden Altersgrenzen zu flankieren, sind eine Verbesserung der Arbeitsbedingungen, eine Reduktion der Arbeitsbelastungen und die Förderung des Arbeits- und Gesundheitsschutzes (Brussig 2015a, 2018). Es ist kontinuierlich zu überprüfen, welchen verändernden Bedingungen der Arbeitswelt (z. B. durch Digitalisierung) den Beschäftigten gegenüberstehen und welchen Unterstützungsbedarf Arbeitgeber und Gewerkschaften (und auch die Rentenversicherungsträger bei der Prävention) leisten können.

Angesichts der aktuellen Entwicklungen auf dem Arbeitsmarkt kann nicht ausgeschlossen werden, dass die Anzahl von arbeitenden Ruheständler*innen aufgrund finanzieller Notwendigkeiten zunehmen wird. Diese Entwicklung würde schließlich zu Legitimationsproblemen der Gesetzlichen Rentenversicherung führen (Bäcker/Jansen/Schmitz 2017). Eine Mindestrente nach langer Erwerbstätigkeit könnte hier ein wirksamer Baustein sein.

Zusammenfassend liegt die Verantwortung einer verlängerten Lebensarbeitszeit nicht ausschließlich auf den Schultern der Beschäftigten, sondern umfasst Akteure auf staatlicher, gewerkschaftlicher und betrieblicher Ebene. Eine (weitere) Zunahme der Erwerbsbeteiligung Älterer und eine Verlängerung der Lebensarbeitszeit können nur dann ermöglicht werden, wenn entsprechende Voraussetzungen kooperativ geschaffen werden.

\section{Literatur}

Bäcker, G. (2012): Erwerbsminderungsrenten: Strukturen, Trends und aktuelle Probleme, IAQ- Altersübergangs-Report 3/2012, Duisburg

Bäcker, G./Jansen, A./Schmitz, J. (2017): Rente erst ab 70? Probleme und Perspektiven des Altersübergangs, IAQ-Forschung 02/2017, Duisburg

BMAS (2016): Verordnungsentwurf des Bundesministeriums für Arbeit und Soziales. Erste Verordnung zur Änderung der Unbilligkeitsverordnung, Berlin

Brussig, M. (2011): Neueinstellungen im Alter: Tragen sie zu verlängerten Erwerbsbiografien bei?, IAQ-Altersübergangs-Report 3/2011, Duisburg

Brussig, M. (2015a): Alter beim Austritt aus sozialversicherungspflichtiger Beschäftigung ist gestiegen. Auch nach dem Ende der sozialversicherungspflichtigen Beschäftigung sind viele Erwerbspersonen noch auf dem Arbeitsmarkt aktiv - mit wachsender Dauer, IAQ-Altersübergangs-Report 1/2015, Duisburg

Brussig, M. (2015b): In die Rente wider Willen?, in: WSI-Mitteilungen 68 (6), S. $407-416$

Brussig, M. (2018): Verlängerte Erwerbsbiografien: Triebkräfte, Grenzen, soziale Ungleichheiten, in: WSI-Mitteilungen 71 (1), S. 12-19 
Brussig, M./Eggers, K. (2014): Langfristige Entwicklungen bei Neueinstellungen von Älteren. Altersungleichheit bei Neueinstellungen geht leicht zurück, Duisburg

Brussig, M./Wojtkowski, S. (2008): Anstieg der Alterserwerbsbeteiligung: Aktuelle demographische Veränderungen geben Rückenwind, IAQ-Altersübergangs-Report $1 / 2008$, Duisburg

Brussig, M./Zink, L. (2018): Erwerbsverlaufsmuster von Männern und Frauen mit Niedrigrenten, IAQ-Altersübergangs-Report 2/2018, Duisburg

Brussig, M./Knuth, M./Mümken, S. (2016): Von der Frühverrentung bis zur Rente mit 67. Der Wandel des Altersübergangs von 1990 bis 2012, Bielefeld

Brussig, M./Knuth, M./Wojtkowski, S. (2009): Altersteilzeit: Zunehmend Beschäftigungsbrücke zum späteren Renteneintritt. Wegfall der Förderung verengt auch den Zugang in nichtgeförderte Altersteilzeit - Nachfolgetarifverträge fehlen, IAQ-Altersübergangs-Report 2/2009, Duisburg

Bundesagentur für Arbeit (2018): Situation von Älteren, Nürnberg

Deutscher Bundestag (2016): Entwurf eines Gesetzes zur Flexibilisierung des Übergangs vom Erwerbsleben in den Ruhestand und zur Stärkung von Prävention und Rehabilitation im Erwerbsleben (Flexirentengesetz)

Dlugosz, S./Stephan, G./Wilke, R. A. (2009): Verkürzte Bezugsdauern für Arbeitslosengeld. Deutliche Effekte auf die Eintritte in Arbeitslosigkeit, IAB-Kurzbericht 30/2009

Dünn, S./Stosberg, R. (2014): Was ändert sich durch das RV-Leistungsverbesserungsgesetz?, in: RVaktuell 7/2014, S. 156-165

Kaboth, A./Brussig, M. (2018): Alterserwerbsbeteiligung in Europa auch in Zeiten der Finanz- und Wirtschaftskrise im Aufschwung?, IAQ-Altersübergangs-Report 1/2018, Duisburg

Kaboth, A./Brussig, M. (2019): Trotz steigender Altersgrenzen überwiegen frühe Rentenzugänge. Aktuelle Entwicklungen im Rentenzugang, IAQ-Altersübergangs-Report 02/2019, Duisburg

Kaboth, A./Brussig, M. (2020): Trotz Alterserwerbsbeteiligung auf Rekordniveau: Mehr Ältere von Arbeitslosigkeit betroffen. Großer Anteil älterer Arbeitsloser bleibt nach wie vor verdeckt, IAQ-Altersübergangs-Report 1/2020, Duisburg

Knuth, M./Kalina, T. (2002): "Vorruhestand" verfestigt die Arbeitslosigkeit. Kalkulierte Arbeitslosigkeit Älterer behindert Aktivierung der Arbeitsmarktpolitik, IAT-Report 2/2002, Gelsenkirchen

Knuth, M./Stegmann, T./Zink, L. (2014): Die Wirkungen des Bundesprogramms "Perspektive 50plus". Chancen für ältere Langzeitarbeitslose, IAQ-Report 1/2014, Duisburg

Lott, M. (2011): WeGebAU für Weiterbildung: präventive Arbeitsmarktpolitik aus betrieblicher Sicht. Ergebnisbericht Modul 2 des Projekts Verschränkung von Arbeitszeit und Weiterbildung im Betrieb - Akteptanz, Potenziale und Wirkungen -, https:/www.boeckler.de/pdf_fof/91236.pdf, (abgerufen am 13.02.2020) 
Mika, T./Lange, J./Stegmann, M. (2014): Erwerbsminderungsrente nach Bezug von ALG II: Auswirkungen der Reformen auf die Versicherungsbiografien, in: WSIMitteilungen 67 (4), S. 277-285

Mümken, S./Brussig, M. (2012): Alterserwerbsbeteiligung in Europa. Deutschland im internationalen Vergleich, IAQ-Altersübergangs-Report 1/2012, Duisburg

Mümken, S./Brussig, M. (2013): Sichtbare Arbeitslosigkeit: Unter den 60- bis 64Jährigen deutlich gestiegen: Reformen zielen auf eine Verlängerung der Erwerbsphasen $\mathrm{ab}$, doch auch die Altersarbeitslosigkeit steigt, IAQ-Altersübergangs-Report 1/2013, Duisburg

OECD (2013): Employment Outlook 2013, http://www.keepeek.com/Digital-AssetManagement/oecd/employment/oecd-employment-outlook-2013_empl_outlook -2013-en\#page55

Schmähl, W. (2011a): Die Entwicklung der Rentenversicherung vom Ende des Zweiten Weltkriegs bis zum Mauerfall (1945-1989), in: Eichenhofer, E./Rische, H./Schmähl, W. (Hrsg.): Handbuch der gesetzlichen Rentenversicherung SGB VI, Köln, S. 33-66

Schmähl, W. (2011b): Von der Ergänzung der gesetzlichen Rentenversicherung zu deren partiellem Ersatz, in: Eichenhofer, E./Rische, H./Schmähl,W. (Hrsg.): Handbuch der gesetzlichen Rentenversicherung - SGB VI, Köln, S. 169-250

Singer, C. (2013): Richtfest. Zehn Jahre Agenda 2010 - ein Besuch auf der Reformbaustelle., Bielefeld, https:/www.wbv.de/journals/zeitschriften/iab-forum/artikel /shop/detail/name/_/0/1/IFO1302W/facet/IFO1302W///////nb/0/category/1058.ht $\mathrm{ml}$

Stein, U./Aricò, F. (2010): Beschäftigungspolitische Krisenreaktion in Deutschland, Italien und dem Vereinigten Königreich, in: WSI-Mitteilungen 71 (11), S. 569576

Walwei, U. (2018): Trends in der Beschäftigung Älterer. Rahmenbedingungen für betriebliche Personalpolitik, in: WSI-Mitteilungen 71 (1), S. 3-11

Wanger, S. (2009): Altersteilzeit. Beliebt, aber nicht zukunftsgerecht, http:// doku.iab.de/kurzber/2009/kb0809.pdf

Ziefle, A. (2017): Der lange Arm der Bildungsexpansion: Die Bedeutung zunehmender elterlicher Bildungsressourcen für die Bildungsbeteiligung von Frauen in Deutschland, in: KZfSS Kölner Zeitschrift für Soziologie und Sozialpsychologie 69 (1), S. 51-77 